\title{
The nature of memory processes underlying recognition judgments in the process dissociation procedure
}

\author{
AXEL BUCHNER \\ University of Trier, Trier, Germany \\ EDGAR ERDFELDER \\ University of Bonn, Bonn, Germany \\ MELANIE C. STEFFENS \\ University of Trier, Trier, Germany \\ and \\ HEIKE MARTENSEN \\ University of Nijmegen, Nijmegen, The Netherlands
}

\begin{abstract}
The hypothesis is tested that the memory processes involved in recognition judgments in the process dissociation procedure are the same as those involved in standard source-monitoring tasks. It is shown how source-monitoring response categories can be mapped onto process dissociation response categories. On the basis of this observation, an experiment was conducted in which it was possible to compare, using a multinomial modeling approach, the parameters representing memory processes in the process dissociation procedure with those involved in source monitoring. For the two different encoding conditions realized, the results are compatible with the hypothesis that the same processes are involved in source monitoring and in recognition judgments in the process dissociation procedure. Implications for the interpretation of the model's parameters are discussed.
\end{abstract}

Jacoby (1991) has proposed an experimental procedure designed to decompose observable task performance into two underlying memory processes, one usually called "conscious" or "controlled," the other usually called "unconscious" or "automatic." This process dissociation procedure is quite popular, and it has been applied successfully to a great variety of experimental situations such as the Stroop task (Lindsay \& Jacoby, 1994), name judgments (Wippich, 1994), fame judgments (e.g., Buchner \& Wippich, 1996; Jennings \& Jacoby, 1993), truth judgments (Begg, Anas, \& Farinacci, 1992), word stem completion (e.g., Debner \& Jacoby, 1994; Toth, Reingold, \& Jacoby, 1994), lexical decision (Vaterrodt-Plünnecke, 1994), memory search (Yonelinas \& Jacoby, 1995), and many other tasks.

This article is concerned with yet another application of the process dissociation procedure. In introducing the

We would like to thank Jürgen Weiler for his assistance with data collection. We also thank Werner Wippich for very valuable comments on an earlier version. The research reported in this article was supported by grants from the Deutsche Forschungsgemeinschaft to A.B. (Bu 945/1-1) and to E.E. (Er 224/1-1). Correspondence concerning this article should be addressed to A. Buchner, FB I-Psychologie, Universität Trier, Universitätsring 15, D-54286 Trier, Germany (e-mail: buchner@ cogpsy.uni-trier.de) procedure, Jacoby (1991) used a recognition task to show how "to separate the effects of recollection from those of familiarity" (p. 527) in recognition performance. Several other researchers have since used the process dissociation procedure in connection with recognition judgments (Buchner, Erdfelder, \& Vaterrodt-Plünnecke, 1995; Hertel \& Milan, 1994; Hintzman \& Curran, 1994; Komatsu, Graf, \& Uttl, 1995; LeCompte, 1995; Verfaellie \& Treadwell, 1993; Yonelinas, 1994). In this particular application, the process dissociation procedure seems to share important features with source-monitoring tasks (cf. Johnson, Hashtroudi, \& Lindsay, 1993), suggesting the possibility that the same memory processes may be involved in the performance of both types of tasks.

Our goal was to investigate this possibility empirically. In what follows, we first describe the feature overlap between source-monitoring tasks and the process dissociation procedure as applied to recognition judgments. More precisely, we show that, in principle, the response categories in a typical source-monitoring task can be mapped onto the response categories of process dissociation experiments using a recognition task. This suggests the possibility that the latter task can be "mimicked" by the former. Subsequently, we present an experiment that was designed to test directly the hypothesis that the feature overlap described in the first section is paralleled by a match in the 
memory processes involved in task performance. To anticipate, our empirical results indicate that recognition judgments in the process dissociation procedure involve essentially the same memory processes as do source discrimination judgments. Moreover, it appears that applications of the process dissociation procedure to recognition tasks may be considered redundant, given standard source-monitoring tasks. We close by arguing that analyses of recognition data in terms of source-monitoring measurement models may be more profitable than analyses based on process dissociation models.

When the process dissociation procedure is applied to a recognition memory paradigm, the minimum requirement is that there be at least two classes of items in the acquisition phase. For instance, subjects could read some words and hear others. Typically, the acquisition phase is divided into two distinct phases, and subjects process one class of items in each of these phases. For instance, they could read words in Phase 1 and hear words in Phase 2. One item class is often considered critical in that subjects' responses to items from this class, but not from the other class, are used for measurement purposes. For the present illustration, we assume that the Phase 1 items are the critical acquisition phase items.

In a subsequent recognition test, subjects respond to the critical Phase 1 items, to Phase 2 items, and to distractor items. The process dissociation procedure requires two different test conditions. In the inclusion test condition, subjects are instructed to call an item old if it was studied in Phase 1 or in Phase 2. Distractors must be called new. In the exclusion test condition, subjects are instructed to judge an item old only if it was presented during Phase 2. In contrast, Phase 1 items must be called new (i.e., they must be "excluded"). The same is true for distractors.

According to Jacoby (1991), consciously controlled and uncontrolled familiarity-related processes contribute to recognition judgments. (We will ignore the possibility of plain guessing for the moment.) The process dissociation procedure, in combination with an appropriate measurement model, is seen as a tool to estimate separately the contributions of the two types of processes. The inclusion test condition represents a facilitation paradigm in which both types of processes may operate "in concert." An item is accepted as old on the basis of a controlled recollection or because of an automatic assessment of its familiarity. The exclusion test condition represents an interference paradigm in which the two process types are said to be put "in opposition." In this condition, subjects try to reject the critical acquisition phase items whenever they can identify them as old. If they nevertheless accept such an item as old, this is assumed to represent the operation of an unconscious and automatic process contributing to item familiarity that could not be counteracted by a controlled recollection.

However, one might also reason that critical acquisition phase items are accepted in the exclusion test condition because, although the item is consciously recollected as an item that had been encountered in the experiment before, one simply confuses the presentation contexts. For instance, one may consciously recollect that a certain word has been encountered in the experiment before, but simply misjudge it as a heard word from Phase 2 when in fact it is a word that was read in Phase 1. This may be conceived of as a classical example of source confusion.

From this perspective, we may wonder whether there is any difference between the memory processes involved in recognition judgments in the process dissociation procedure and those involved in typical source-monitoring tasks. In fact, if we assume that there is no difference in the processes contributing to performance in both types of tasks, it is easy to show how we may recover old and new responses for a process dissociation design from sourcemonitoring data. This is illustrated in Table 1 .

For the present purposes, it is sufficient to consider responses to Phase 1 words and to distractors, and it seems convenient to continue assuming that words have been read in Phase 1 and heard in Phase 2. The first row in Table 1 illustrates that whenever subjects would correctly recognize a Phase 1 word as a read word, they would respond "Phase 1" in a source-monitoring task, "old" in the inclusion condition of the process dissociation procedure, and "new" in the exclusion condition of the process dissociation procedure (because Phase 1 words must be "excluded"). If they recollected a read word but misjudged its source and classified it as a heard word, they would respond "Phase 2" in a source-monitoring task, and "old" in both the inclusion and exclusion conditions of the process dissociation procedure. Finally, if subjects erroneously thought that a read word was a distractor, they would respond "new" in a source-monitoring task as well as in both test conditions of the process dissociation procedure.

The same mapping applies to judgments about distractors. If subjects falsely believed that a distractor was a word they had read in Phase 1, they would respond "Phase 1" in a source-monitoring task, "old" in the inclusion and "new" in the exclusion test conditions of the process dissociation procedure. If they mistook it for a Phase 2 word, they would respond "Phase 2" in a source-monitoring task and "old" in both the inclusion and the exclusion test conditions of the process dissociation procedure. Finally, if subjects correctly concluded that they had never encountered a distractor word in the experiment, they would respond "new" in a source-monitoring task as

Table 1

Illustration of How Response Categories in a Source-Monitoring Task Can Be Mapped Onto the Response Categories in the Two Test Conditions in the Process Dissociation Procedure for a Recognition Task

\begin{tabular}{|c|c|c|c|}
\hline \multirow[b]{2}{*}{ Item Type } & \multirow{2}{*}{$\begin{array}{l}\text { Source- } \\
\text { Monitoring } \\
\text { Response }\end{array}$} & \multicolumn{2}{|c|}{ Process Dissociation Response } \\
\hline & & $\begin{array}{l}\text { Inclusion } \\
\text { Condition }\end{array}$ & $\begin{array}{l}\text { Exclusion } \\
\text { Condition }\end{array}$ \\
\hline \multirow[t]{3}{*}{ Phase 1 word } & Phase 1 & old & new \\
\hline & Phase 2 & old & old \\
\hline & new & new & new \\
\hline \multirow[t]{3}{*}{ Distractor word } & Phase 1 & old & ne: \\
\hline & Phase 2 & old & old \\
\hline & new & new & new \\
\hline
\end{tabular}


well as in both test conditions of the process dissociation procedure.

In order to test whether this reasoning is correct, we may take the response frequencies obtained in a sourcemonitoring task, use the mapping rules illustrated in Table 1 to aggregate them, and thus reconstruct the response frequencies as they would result from a process dissociation experiment. We can then use these reconstructed frequencies to estimate the parameters representing the memory processes involved. If the assumptions underlying the preceding analysis are correct, the memory parameters as estimated from the reconstructed response frequencies should be equal to the memory parameters as estimated from genuine process dissociation response frequencies.

The following experiment was designed to test this prediction. It was modeled after that presented by Jacoby (1991) to introduce the process dissociation procedure. In Phase 1, the critical acquisition phase of our experiment, subjects read a list of words and solved anagrams. In Phase 2, they memorized a list of heard words for a later recognition test. One half of the subjects were then transferred to the typical process dissociation test procedure, in which they received either inclusion or exclusion instructions and the response categories were old and $n e w$, as described above. The second half of the subjects received standard source-monitoring instructions, according to which words had to be classified as Phase 1 , Phase 2, or new. These subjects were again divided randomly into two groups. The responses from one group were mapped, according to the mapping rules illustrated in Table 1, onto the process dissociation response categories as if they had been obtained in an inclusion test condition, whereas the responses from the other group were mapped onto the process dissociation response categories as if they had been obtained in an exclusion test condition.

For the process dissociation and the source-monitoring groups, the parameters representing the memory processes involved were estimated from the observed and the reconstructed responses, respectively. We took a multinomial modeling approach to estimating the model parameters (e.g., Hu \& Batchelder, 1994; Riefer \& Batchelder, 1988), and the measurement model underlying the parameter estimates was the extended model suggested by Buchner et al. (1995) for the process dissociation procedure. The model is illustrated in Figure 1 in the form of a general processing tree model. It differs from Jacoby's (1991) original suggestion, in that it includes guessing parameters to take possible response biases into account. When the extended model is applied to recognition judgments, parameter $c$ denotes the unconditional probability of recollecting a critical acquisition phase item. Parameter $u_{\mathrm{c}^{+}}$denotes the conditional probability of uncontrolled processes resulting in a feeling of familiarity, given that a critical acquisition phase item has already been recollected. This parameter can be ignored for the present purposes because it does not influence subjects' responses. ${ }^{1}$ Parameter $u_{\mathrm{c}-}$ represents the conditional probability of uncontrolled pro- cesses leading to a cognitive state in which a critical acquisition phase item is accepted, given that it has not been recollected. Finally, parameters $g_{\mathrm{i}}$ and $g_{\mathrm{e}}$ represent the probabilities of guessing that an item is old, given that it is neither recollected nor familiar in the inclusion and exclusion test conditions, respectively.

Briefly, the model assumes that old responses to Phase 1 items in the inclusion test condition can result (1) if the item is recollected, which occurs with probability $c ;(2)$ if the item is not recollected but appears familiar, which occurs with probability $(1-c) u_{c-}$; or (3) if the item is neither recollected nor seems familiar but it is guessed that the item is from Phase 1 , which occurs with probability $(1-c)\left(1-u_{\mathrm{c}^{-}}\right) g_{\mathrm{i}}$. Distractors cannot be recollected and cannot seem familiar. Thus, old responses to distractors are the result of guessing that an item is old, which occurs with probability $g_{i}$. The model assumes further that old responses to Phase 1 items in the exclusion test condition can result only if the item is not recollected but appears familiar, which occurs with probability $(1-c) u_{c-}$, or because of guessing when an item neither is recollected nor seems familiar; this occurs with probability $(1-c)\left(1-u_{\mathrm{c}-}\right) g_{\mathrm{e}}$. Old responses to distractors which cannot be recollected and cannot seem familiar are the result of guessing that an item is old, which occurs with probability $g_{\mathrm{e}}$. For the demonstration that the extended model provides for identifiable parameters representing the memory processes (i.e., $c$ and $u_{\mathrm{c}-}$ ) as well as the guessing processes (i.e., $g_{\mathrm{i}}$ and $g_{\mathrm{e}}$ ), and for further details, see Buchner et al. (1995).

\section{METHOD}

\section{Subjects}

The subjects were 36 female and 28 male persons, 39 of whom were students at the University of Trier who either volunteered or received course credit for participating in the experiment. The remaining 25 subjects were recruited from a pool of nonstudent volunteers. The subjects ranged in age from 18 to 57 years $(M=27.22$, $S D=5.81$ ). They were assigned at random to one of the following four experimental groups: the process dissociation, inclusion test group; the process dissociation, exclusion test group; the sourcemonitoring, inclusion test group; and the source-monitoring, exclusion test group.

\section{Stimuli and Apparatus}

A set of 100 words was selected from a pool of words scaled for word frequency and concreteness (Baschek, Bredenkamp, Oehrle, \& Wippich, 1977). All words were five-letter German nouns in singular form. These were assigned to four lists of 25 words each as follows. First, all words were ranked for both word frequency and concreteness. Then, the first four words of this list were each randomly assigned to one of the four different lists. This randomization procedure was repeated for all remaining quadruples of words so that the final lists were parallel with respect to both word frequency and concreteness.

Words from one of these four lists served as words to be read in Phase 1, words from a second list were presented as anagrams in Phase 1, words from a third list were to be heard in Phase 2, and words from the fourth list were added as distractor words in the recognition memory test in Phase 3 of the experiment. The four lists of 25 words were rotated through the four presentation conditions (read, anagram, hear, and distractor) so that each set of words was 

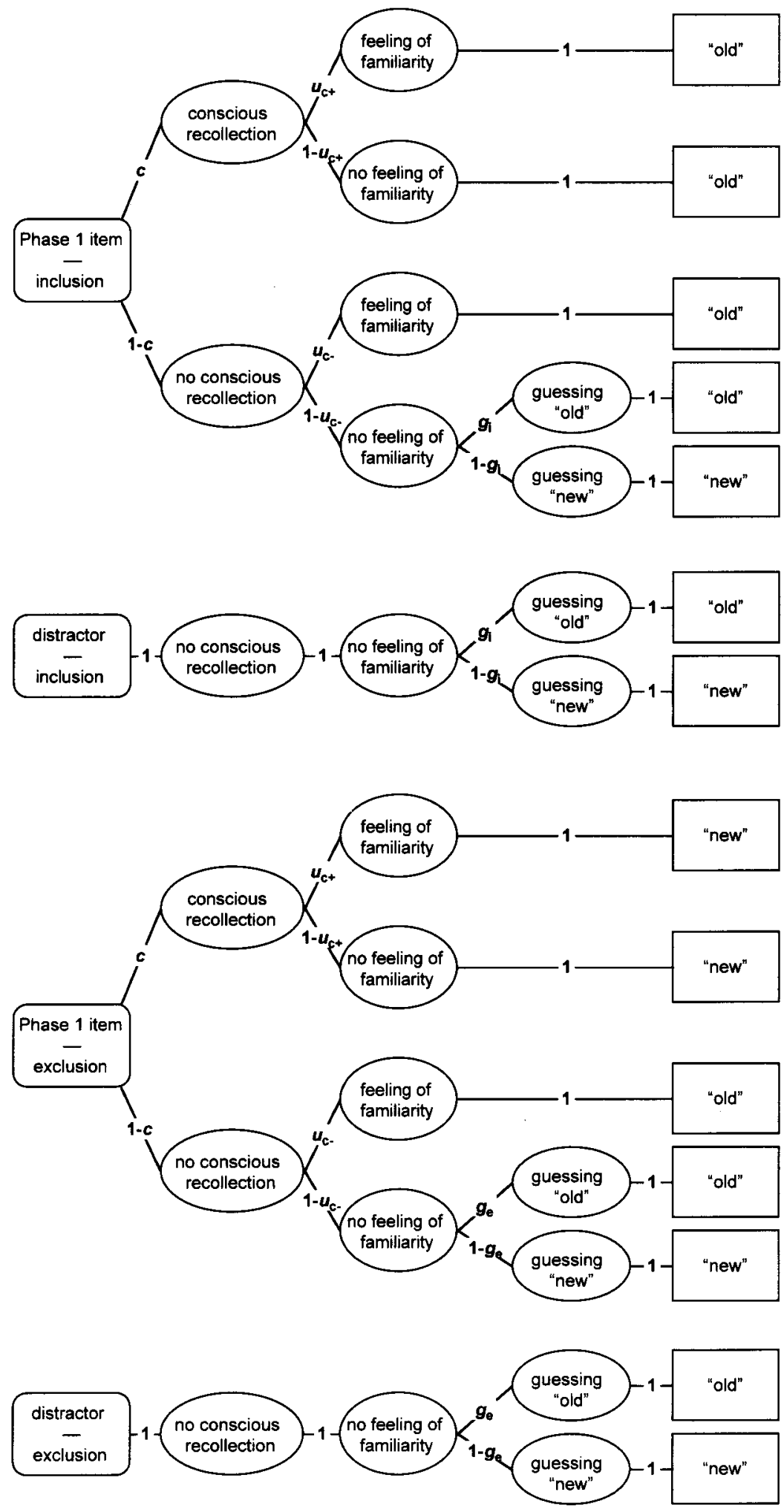

Figure 1. Tree diagram for the extended measurement model for the process dissociation procedure. Events depicted in rectangles represent observable test conditions (rounded rectangles, left side) and observable responses (ordinary rectangles, right side). Events depicted in ovals represent unobservable cognitive states. The parameters attached to the branches denote the probabilities for certain cognitive events. From "Toward Unbiased Measurement of Conscious and Unconscious Memory Processes Within the Process Dissociation Framework," by A. Buchner, E. Erdfelder, and B. Vaterrodt-Plünnecke, 1995, Journal of Experimental Psychology: General, 124, p. 144. Copyright 1995 by the American Psychological Association. Reprinted with permission. 
used in each of the presentation conditions, effectively constituting four different sets of words.

The anagrams were constructed as in Jacoby (1991); that is, the second and fourth letters in each word were kept in their proper positions and underlined to make this transparent to the subjects. The remaining letters were rearranged so that each letter occupied a position different from its original position.

Words to be heard were spoken in a male voice. They were recorded and digitized individually on a Macintosh AV computer. During the experiment, the words were presented binaurally through stereo headphones plugged directly into the computer. The sound level was adjusted to be at about $72 \pm 5 \mathrm{~dB}(\mathrm{~A})$.

The list of words for the final recognition test was composed of all words from all four word lists and thus comprised a total of 100 words. The sequence of words to be read and anagrams to be solved in Phase 1 was randomized for every subject, as were the sequence of words to be heard during Phase 2 and the sequence of test words in Phase 3.

\section{Procedure}

The entire experiment lasted, on the average, $28 \mathrm{~min}(S D=7 \mathrm{~min})$ The subjects were tested one at a time. In Phase 1 of the experiment, the subjects were instructed that their task was to read words presented on the computer screen and to write them down as fast as possible. They were led to believe that the purpose of the experiment was to evaluate the legibility of words in different fonts on the computer screen. The recognition test at the end of the experiment was not mentioned. It was explained that some words would appear as anagrams and that the task was to form the correct word from each anagram and write it down as fast as possible. The subjects learned that the second and fourth letters in each anagram were underlined and in their correct positions so that only the remaining three letters had to be rearranged to generate the correct word. It was emphasized that response times were being measured, and that the written word lists would later be evaluated for accuracy.

The subjects were allowed a maximum of $30 \mathrm{sec}$ to generate a word from an anagram. After that period, the correct solution was shown. The subjects were told to write down the correct word and then to initiate the next trial by pressing the space bar on the keyboard.

At the beginning of Phase 2, the subjects were informed that they would hear a list of words that they were to write down and to memorize for a later recognition test. Words were presented at a rate of $4 \mathrm{sec} /$ word.

In Phase 3, each test word was presented at the center of the computer screen for the recognition test, and the appropriate response buttons appeared below each word. The subjects in the process dissociation, inclusion test group were told to press, with the computer mouse, the old button if they had heard, read, or generated the word from an anagram. They were asked to press the new button if the word was new. The subjects in the process dissociation, exclusion test group were to press the old button only if they had heard the word, and to press the new button for all other words, including the words that they had read or solved as anagrams. The subjects in both source-monitoring groups received identical instructions: They were told to press the Phase $I$ button if they remembered having solved that word as an anagram or if they remembered having read it in Phase 1 . They were to press the Phase 2 button if they remembered having heard the word in Phase 2. Finally, the new button was to be pressed for new words

Feedback about the correctness of the subjects' judgments was given every 10 trials. For each correct recognition judgment, subjects received one credit. For each incorrect recognition judgment, one credit was deducted from the current score. As an incentive, the subjects were told that, at the end of the experiment, their names would be entered into a high score list if their recognition performance was good enough, and a material award was promised to the best subjects. After the experiment, all subjects were debriefed and the purpose of the experiment was described.

\section{Design}

The dependent variable was the subjects' recognition performance. Independent variables were (1) test procedure (process dissociation vs. source monitoring; between subjects) and (2) test condition (inclusion vs. exclusion; between subjects). There were 16 subjects in each of the treatment combinations of this $2 \times 2$ design. Within each treatment combination, equal numbers of subjects (4) were assigned to each of the four different word sets.

Each of the 64 subjects contributed 25 responses to the read (or anagram) words and 25 responses to the distractor words, resulting in $N=64 \cdot 50=3,200$ for the model test. We decided that we wanted to detect "small effects" ( $w=0.1$ for $\chi^{2}$ tests; cf. Cohen, 1977 ) in the deviations between the actual response probabilities and the response probabilities as predicted by the restricted model defining the null hypothesis. Further, we thought it important to consider the Type I and Type II error probabilities equally serious. Given these premises and two degrees of freedom for the model test (see below), a compromise power analysis (Erdfelder, Faul, \& Buchner, 1996) suggested the choosing of error probabilities of $\alpha=\beta=$ .0055 (which implies $\chi^{2}$ (crit) $\left.=10.4211\right){ }^{2}$ Thus, the power to detect even a "small" effect in the model test reported below is $1-\beta=$ 9945. Note, however, that although we consider $\chi^{2}$ (crit) $=10.4211$ to be a reasonable critical value for the model tests reported in this article, our conclusions do not depend on choosing this particular critical value. Conventional levels of significance such as $\alpha=.05$ would have led to the same conclusions for each of the tests reported below.

\section{RESULTS}

The subjects had no difficulty in writing down the tobe-read words presented on the computer screen $(99.6 \%$ correct). It took them about $4.1 \mathrm{sec}$ for one word $(S D=$ $2.0 \mathrm{sec}$ ). The subjects solved $81.0 \%$ of the anagrams correctly within the given time limit of $30 \mathrm{sec}$, and it took them, on the average, $10.6 \mathrm{sec}(S D=2.5 \mathrm{sec})$ to solve one anagram. For $9.6 \%$ of the anagrams, the subjects' attempts to form the correct word exceeded the $30-\mathrm{sec}$ limit after which they were shown the correct solution and asked to write it down. For the remaining $9.4 \%$ of the anagrams, the subjects produced an incorrect solution. Finally, $99 \%$ of the words heard in Phase 2 were written down correctly.

We tested whether the subjects' acquisition phase performance varied as a function of test procedure, test condition, or word set. With respect to the number of read words written down correctly, we found no statistically significant main effects of test procedure and of test condition, and no interaction between these independent variables [all $F \mathrm{~s}(1,48)<0.75$ ], as well as no other interaction between any of the independent variables and no statistically significant main effect of word set [all $F_{\mathrm{s}}(3,48)<$ $1.50]$. The same was true for the number of correct anagram solutions [all $F_{\mathrm{s}}(1,48)<1.20$ and all $F_{\mathrm{s}}(3,48)<1.59$ ] and for the number of heard words written down correctly [all $F_{\mathrm{s}}(1,48)<3.00$ and all $F_{\mathrm{s}}(3,48)<2.68$ ].

Table 2 displays the frequencies of responding "old" and "new" to the words read or solved as anagrams in Phase 1 and to the distractor words for the process dis- 
sociation groups. Table 2 also shows the frequencies that were reconstructed according to the mapping rules illustrated in Table 1 from the source-monitoring data for the source-monitoring groups. The original source-monitoring frequency data are presented in the Appendix.

In order to evaluate statistically our hypothesis that the memory processes involved in recognition judgments in the process dissociation procedure are the same as those involved in the source-monitoring task, we used a multinomial modeling approach and performed the following steps. First, we used the unrestricted extended measurement model for the process dissociation procedure (Buchner et al., 1995) to estimate, for the read words, parameter $c$, which is assumed to represent recollection processes, parameter $u_{\mathrm{c}-}$, which is assumed to represent automatic familiarity-related processes, and the guessing parameters $g_{\mathrm{i}}$ and $g_{\mathrm{e}}$. The upper panel of Figure 2 displays these parameter estimates, together with the $95 \%$ confidence intervals.

Comparing the parameter estimates based on the process dissociation response frequencies with the estimates based on the frequencies reconstructed from the sourcemonitoring task, we find that both $\hat{c}$ and $\hat{u}_{\mathrm{c}-}$ differ very little between conditions. In fact, the confidence intervals overlap almost completely. In a second step, we performed goodness-of-fit tests for a model in which we implemented equality restrictions on the parameters representing controlled and automatic memory processes. More precisely, the restrictions imposed were that $c$ (process dissociation) $=c$ (source monitoring) and that $u_{\mathrm{c}-}$ (process dissociation) $=u_{\mathrm{c}-}$ (source monitoring). The two restrictions on the unrestricted model yield two degrees of freedom for the model test. The likelihood-ratio goodness-of-fit statistic indicates that the data are compatible with the restricted model $\left[G^{2}(2)=1.00\right] .^{3}$

Similarly, when looking at the parameter estimates for the anagram data (displayed in the lower panel of Figure 2), we find that both $\hat{c}$ and $\hat{u}_{\mathrm{c}}$ do not differ much between conditions. As for the read words, we performed goodness-of-fit tests for the model in which we implemented the restrictions that $c$ (process dissociation $)=$ $c$ (source monitoring) and that $u_{\mathrm{c}-}$ (process dissociation) $=u_{\mathrm{c}-}$ (source monitoring). The likelihood-ratio goodness-

Table 2

Frequencies of "Old" and "New" Responses Which Were Either Obtained Directly (Process Dissociation Condition) or Derived From Source Discrimination Data According to the Mapping Rules of Table 1 (Source-Monitoring Condition)

\begin{tabular}{|c|c|c|c|c|c|c|}
\hline \multirow{3}{*}{$\begin{array}{c}\text { Test } \\
\text { Condition }\end{array}$} & \multicolumn{4}{|c|}{ Phase 1 Words } & & \\
\hline & \multicolumn{2}{|c|}{ Read Words } & \multicolumn{2}{|c|}{ Anagram Words } & \multicolumn{2}{|c|}{ Distractor Words } \\
\hline & Old & New & Old & New & Old & New \\
\hline \multicolumn{7}{|c|}{ Process Dissociation Condition } \\
\hline Inclusion & 208 & 192 & 315 & 85 & 64 & 336 \\
\hline Exclusion & 78 & 322 & 57 & 343 & 25 & 375 \\
\hline \multicolumn{7}{|c|}{ Source-Monitoring Condition } \\
\hline Inclusion* & 211 & 189 & 339 & 61 & 76 & 324 \\
\hline Exclusiont & 69 & 331 & 51 & 349 & 29 & 371 \\
\hline
\end{tabular}

*Phase $I$ and Phase 2 responses were counted as old responses. ${ }^{+}$Only Phase 2 responses were counted as old responses. of-fit statistic indicates, again, that the data are compatible with the restricted model $\left[G^{2}(2)=4.16\right]$. In other words, the anagram data essentially replicate the read data. ${ }^{4}$

In the statistical tests reported so far, the restrictions were imposed only on the parameters reflecting memory processes (i.e., $c$ and $u_{c_{-}}$) and not on those reflecting response strategies (i.e., $g_{\mathrm{i}}$ and $g_{\mathrm{e}}$ ), because the former parameters reflect our original substantive hypothesis that the memory processes involved in recognition judgments in the process dissociation procedure are essentially the same as those involved in source monitoring. According to this hypothesis, response strategies such as guessing in situations of uncertainty may or may not be equivalent in the process dissociation procedure and in the sourcemonitoring task.

However, when one looks at the response frequencies displayed in Table 2, it is obvious that the frequencies obtained in the classical process dissociation procedure and those reconstructed from the source-monitoring design are relatively close. This pattern suggests that a hypothesis which is even stronger than our original hypothesis might be tenable--namely, that the entire recognition behavior in the process dissociation procedure is predictable from source-monitoring behavior. To evaluate this supposition, we tested the $H_{0}$ that the response probabilities underlying the upper half of Table 2 (i.e., the original process dissociation responses) are identical to those underlying the lower half of Table 2 (i.e., the process dissociation responses as predicted from source-monitoring responses) using a chi square test. The $\log$-likelihood $G^{2}$ for the statistical test of this hypothesis is $G^{2}(6)=7.52$, which is not significant, given the critical value $\chi^{2}(6)=$ $12.59, \alpha=.05$. This result is consistent with the substantive hypothesis that both memory and judgment processes did not differ between the process dissociation and source-monitoring tasks. Even more importantly, this conclusion does not depend on the model used to derive the measures for the memory and judgment processes. Given our data, any measurement model must lead to the conclusion that all types of cognitive processes can be considered equivalent in source-monitoring tasks and in the process dissociation procedure when applied to recognition judgments, simply because the judgments in the latter application of the process dissociation procedure are predictable from source-monitoring judgments.

\section{DISCUSSION}

The primary goal of the research reported in this article was to test the hypothesis that the memory processes involved in recognition judgments in the process dissociation procedure are the same as those involved in standard source-monitoring tasks. The results that we obtained for words read in Phase 1 are clearly compatible with this hypothesis. The anagram results replicate this finding.

However, it turned out that our data are compatible with an even stronger hypothesis. The original process dissociation responses were found to be predictable from the pro- 

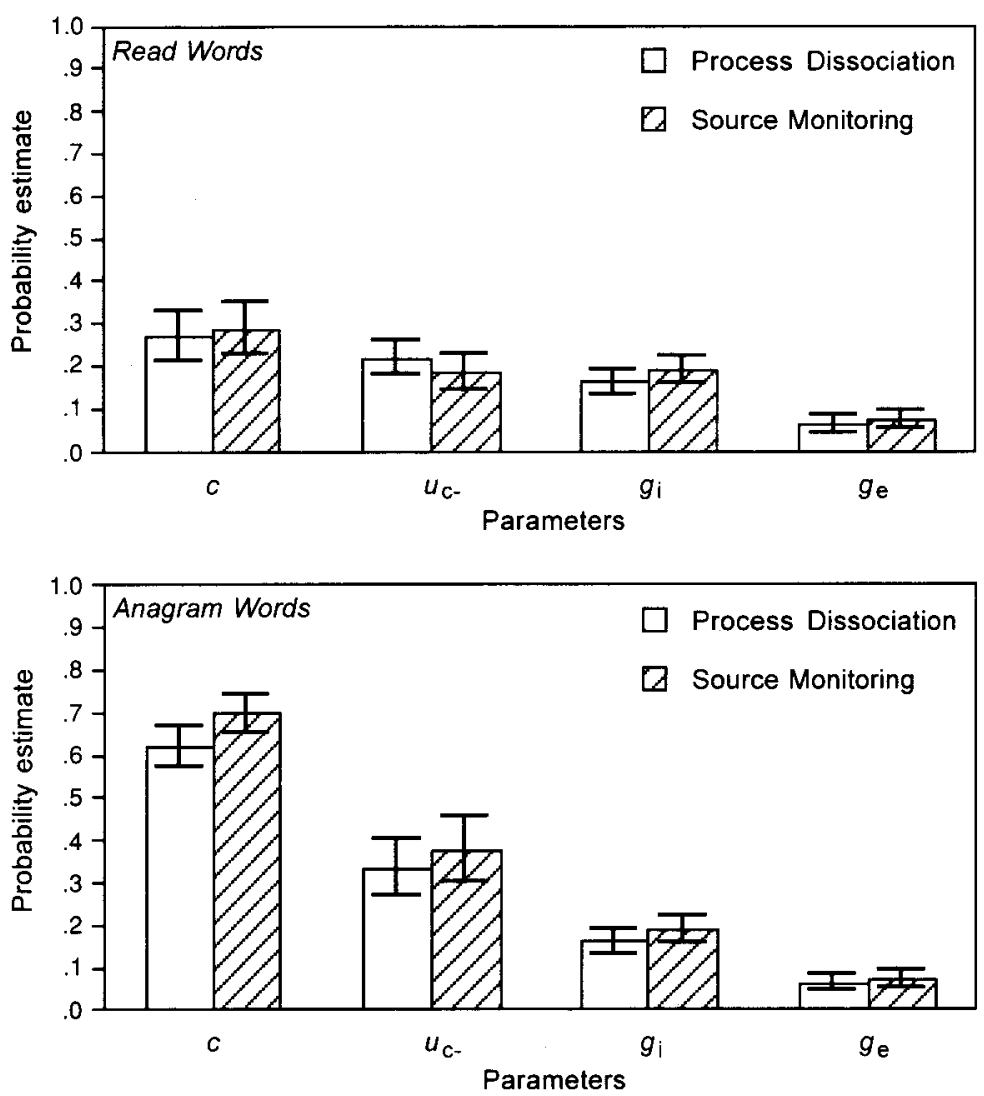

Figure 2. Estimates of parameters representing controlled, uncontrolled, and guessing processes for the read words (upper panel) and for the anagrams (lower panel) as a function of whether the frequencies underlying the parameter estimates were based on process dissociation or source-monitoring conditions. The estimates for the guessing parameters are identical for the read words and for the anagrams. The error bars represent the $95 \%$ confidence intervals.

cess dissociation responses reconstructed from sourcemonitoring responses, which is exactly what one would expect if not the memory processes alone, but both memory and judgment processes did not differ between the process dissociation and the source-monitoring tasks.

This means, at least, that the inclusion-exclusion instructional manipulation, which has been criticized as potentially being too difficult (Graf \& Komatsu, 1994; Richardson-Klavehn, Gardiner, \& Java, 1995), is not necessary in order to arrive at parameter estimates for the memory processes to be dissociated. All we need is a standard source-monitoring task which quite naturally delivers the response frequencies called for by a process dissociation measurement model. Note, however, that this applies primarily to recognition judgments, because applications of the process dissociation procedure to other judgment tasks typically do not require different learning contexts or sources (but see, e.g., Begg et al., 1992).

In an interesting article, Roediger and McDermott (1994) seem to go one step further when they argue that there may be a "problem of source memory affecting the components derived from the process dissociation pro- cedure" (p. 287). The present analysis supports their argument empirically. The subjects responding according to standard source-monitoring instructions showed a performance pattern that could be mapped onto the performance exhibited by subjects following process dissociation instructions, and the estimates representing the memory processes involved in recognition performance in both conditions matched quite well. This is compatible with the conclusion that what subjects do in the process dissociation procedure is essentially source monitoring. If that was the case, however, the parameters representing the memory processes to be dissociated would have to be interpreted differently. For instance, in support of their arguments against an uncritical application of the process dissociation procedure, Roediger and McDermott quote a personal communication with F. I. M. Craik raising exactly this point and arguing that the parameter intended to represent conscious item recollection in the process dissociation procedure "does not measure direct recollection of items. It measures recollection of the presentation context" (p. 287). A similar intuition may be that perhaps an interpretation of the parameter thought to 
capture automatic, unconscious influences of familiarity might be source confusion despite conscious item recollection.

However, if the memory parameters in the process dissociation procedure simply captured certain aspects of source memory, the idea suggests itself that it could be more profitable to utilize measurement models that have been designed specifically to decompose performance on source-monitoring tasks into component processes. For instance, Batchelder and Riefer (1990) have presented a whole family of two-source multinomial processing models for the standard source-monitoring task. Bayen, Murnane, and Erdfelder (1996) have successfully evaluated two-high threshold alternatives to Batchelder and Riefer's one-high threshold models, and Riefer, $\mathrm{Hu}$, and Batchelder (1994) have demonstrated the usefulness of a three-source generalization of their model family for sophisticated analyses of subjects' response strategies. All of these models allow memory performance to be decomposed into measures for the separate component processes underlying source memory, item memory, source bias, and item bias.

Can we clarify analytically, rather than intuitively, how the parameters of source memory models relate to the parameters representing recollection and familiarity as derived from the process dissociation procedure? We will restrict our analysis to the source-monitoring model developed by Batchelder and Riefer (1990), which appears to be the most frequently used measurement model in source-monitoring research (e.g., Johnson, Kounios, $\&$ Reeder, 1994). First, we need to map the sourcemonitoring response categories onto the old and new responses for the inclusion and the exclusion condition according to the rules illustrated in Table 1. For simplicity, we assume that source bias does not depend on whether or not an item is recognized as old. Given this, the equations of Batchelder and Riefer's source-monitoring model imply that the probabilities of old responses to (1) items from Phase 1 in the inclusion test, $p_{1 \mathrm{i}}$, (2) distractor items in the inclusion test, $p_{\mathrm{di}},(3)$ items from Phase 1 in the exclusion test, $p_{1 \mathrm{e}}$, and (4) distractor items in the exclusion test, $p_{\mathrm{de}}$, can be formulated as follows:

$$
\begin{aligned}
& p_{1 \mathrm{i}}=1-\left(1-D_{1}\right)(1-b), \\
& p_{\mathrm{di}}=b, \\
& p_{1 \mathrm{l}}=D_{1}\left(1-d_{1}\right)(1-g)+\left(1-D_{1}\right) b(1-g),
\end{aligned}
$$

and

$$
p_{\mathrm{de}}=b(1-g) \text {, }
$$

where $D_{1}$ is defined as the probability of detecting correctly that a Phase 1 word is an old word (item memory), $d_{1}$ is defined as the probability of discriminating the source of the Phase 1 words (source memory), $b$ is defined as the probability of classifying a nondetected word as old (item bias), and $g$ is defined as the probability of responding "Phase 1" to a word that has been classified as an old word but of which the source is unknown (source bias) (for details, see Batchelder \& Riefer, 1990, p. 551).
We now simply write the same response probabilities in terms of the extended model for the process dissociation procedure (see Buchner et al., 1995, Equations 9-12), set these four model equations of the extended model equal to their corresponding equations listed above, and solve for the parameters of the extended model to obtain

$$
\begin{aligned}
c & =D_{1}\left[d_{1}+\left(1-d_{1}\right) g\right], \\
u_{\mathrm{c}-} & =\left[D_{1}\left(d_{1}-1\right)(g-1)\right] /\left\{1-D_{1}\left[d_{1}+\left(1-d_{1}\right) g\right]\right\}, \\
g_{\mathrm{i}} & =b,
\end{aligned}
$$

and

$$
g_{\mathrm{e}}=b(1-g) \text {. }
$$

Unfortunately both $c$ and $u_{\mathrm{c}-}$ turn out to be rather complicated functions of item memory, source memory, and source bias. From this perspective, there is no simple interpretation of either $c$ or $u_{\mathrm{c}-}$ in terms of item memory and source memory parameters.

Interestingly, however, matters become somewhat simpler if we adopt the redundancy variant of the extended measurement model family, which assumes that recollection always implies a feeling of familiarity (cf. Buchner et al., 1995; Joordens \& Merikle, 1993). It is useful, for our purpose, to reparameterize the redundancy variant of the extended model so that (1) parameter $u$ denotes the unconditional probability of uncontrolled processes leading to a cognitive state in which a word is accepted on the basis of its familiarity, (2) parameter $c_{u^{+}}$represents the conditional probability of recollection given a feeling of familiarity, and (3) parameters $g_{\mathrm{i}}$ and $g_{\mathrm{e}}$ represent, as before, the probabilities of guessing that a word is old, given that it is neither recollected nor familiar in the inclusion and in the exclusion test conditions, respectively. The model equations for this reparameterized model variant are:

$$
\begin{aligned}
& p_{1 \mathrm{i}}=u+(1-u) g_{\mathrm{i}}, \\
& p_{\mathrm{di}}=g_{\mathrm{i}}, \\
& p_{\mathrm{le}}=u\left(1-c_{\mathrm{u}+}\right)+(1-u) g_{\mathrm{e}},
\end{aligned}
$$

and

$$
p_{\mathrm{de}}=g_{\mathrm{e}} \text {. }
$$

Setting these four equations equal to the corresponding model equations as implied by Batchelder and Riefer's (1990) source monitoring model (see Equations 1-4 above) and solving for $u, c_{\mathrm{u}}, g_{\mathrm{i}}$, and $g_{\mathrm{e}}$ yields

$$
\begin{aligned}
u & =D_{1}, \\
c_{\mathrm{u}+} & =d_{1}+g\left(1-d_{1}\right), \\
g_{\mathrm{i}} & =b,
\end{aligned}
$$

and

$$
g_{\mathrm{e}}=b(1-g) \text {. }
$$

Thus, according to the redundancy variant of the extended measurement model for the process dissociation 
procedure, the unconditional probability of an unconscious process on the basis of familiarity is just the item memory parameter of Batchelder and Riefer's (1990) sourcemonitoring model, and the conditional probability of recollection, given a feeling of familiarity, is just the source memory parameter, provided that subjects do not arrive at correct source judgments by guessing (i.e., $g=0$ ).

We may conclude from this analysis that the parameters of at least one submodel of the extended measurement model family as discussed by Buchner et al. (1995) correspond quite closely to the parameters representing memory processes in the source-monitoring model of Batchelder and Riefer (1990). Moreover, one can say that this process dissociation model variant provides no substantive information over and above the information already provided by an analysis in terms of Batchelder and Riefer's model.

In a nutshell, then, our central conclusion is that recognition judgments in the process dissociation procedure involve essentially the same memory processes as does performance in a standard source-monitoring task for which source-monitoring models deliver measures of item memory, source memory, and various response biases. These parameters have clear-cut psychological interpretations because they are integrated into a network of psychological hypotheses and theories (Batchelder \& Riefer, 1990; Bayen et al., 1996; Johnson et al., 1994; Riefer et al., 1994). In addition, a large number of empirical results have become available during the past two decades which help clarify the scope of these concepts (see Johnson et al., 1993).

In contrast, discussions such as the one raised by Roediger and McDermott (1994) and the present results provide evidence that the interpretation of recollection and familiarity within the process dissociation procedure is less clear-cut. Furthermore, depending on the measurement model used, these constructs can have vastly different meanings, some of which can be shown to reduce to the meaning of item memory and (biased) source memory in terms of Batchelder and Riefer's (1990) model.

Thus, on the basis of our present empirical and analytical results we suggest that one use standard sourcemonitoring tasks instead of recognition tasks in the process dissociation procedure. The latter type of memory task can be considered redundant, given the first type of task. Moreover, and irrespective of which of these two types of tasks is used, data analyses in terms of the sourcemonitoring parameters for item memory, source memory, item bias, and source bias seem to be more profitable than analyses based on process dissociation parameters for recollection and familiarity.

It is important to note, however, that these recommendations only concern applications of the process dissociation procedure to recognition judgments. As stated in the introduction of this article, applications of Jacoby's (1991) procedure to other judgments proved to be very successful, and currently we can see no reason to re- place these applications with other types of memory tasks.

\section{REFERENCES}

Baschek, I. L., Bredenkamp, J., Oehrle, B., \& WiPPich, W. (1977), Bestimmung der Bildhaftigkeit (I), Konkretheit (C) und der Bedeutungshaltigkeit $\left(\mathrm{m}^{* *} 1\right)$ von 800 Substantiven [Determination of imagery, concreteness, and meaningfulness of 800 nouns]. Zeitschrift für Experimentelle und Angewandte Psychologie, 24, 353-396.

Batchelder, W. H., \& Riefer, D. M. (1990). Multinomial processing models of source monitoring. Psychological Review, 97, 548-564

Bayen, U. J., Murnane, K., \& Erdfelder, E. (1996). Source discrimination, item detection, and multinomial models of source monitoring. Journal of Experimental Psychology: Learning, Memory, \& Cognition, 22, 197-215.

Begg, I. M., Anas, A., \& Farinacci, S. (1992). Dissociation of processes in belief: Source recollection, statement familiarity, and the illusion of truth. Journal of Experimental Psychology: General, 121, 446-458.

BUChNer, A., ERdfelder, E., \& VaterRodt-PlünNecke, B. (1995) Toward unbiased measurement of conscious and unconscious memory processes within the process dissociation framework. Journal of Experimental Psychology: General, 124, 137-160.

BuchNer, A., FAUL, F., \& Erdfelder, E. (1992). G Power: A priori post-hoc, and compromise power analyses for the Macintosh [Computer program]. Bonn: Bonn University, Department of Psychology.

BUCHNER, A., \& WIPPICH, W. (1996). Unconscious gender bias in fame judgments? Consciousness \& Cognition, 5, 197-220.

COHEN, J. (1977). Statistical power analysis for the behavioral sciences (rev. ed.). Hillsdale, NJ: Erlbaum.

DeBner, J. A., \& JACOBY, L. L. (1994). Unconscious perception: Attention, awareness, and control. Journal of Experimental Psychology. Learning, Memory, \& Cognition, 20, 304-317.

Erdfelder, E., Faul, F., \& Buchner, A. (1996). GPower: A general power analysis program. Behavior Research Methods, Instruments, \& Computers, 28, 1-11.

Graf, P., \& Komatsu, S.-I. (1994). Process-dissociation procedure Handle with caution! European Journal of Cognitive Psychology, 6 , 113-129.

HerTEL, P. T., \& Milan, S. (1994). Depressive deficits in recognition: Dissociation of recollection and familiarity. Journal of Abnormal Psychology, 103, 736-742.

HintzMan, D. L., \& CuRRan, T. (1994). Retrieval dynamics of recognition and frequency judgments: Evidence for separate processes of familiarity and recall. Journal of Memory \& Language, 33, 1-18

$\mathrm{Hu}, \mathrm{X}$. (1993). Statistical inference program for multinomial binary tree models [Computer program]. Irvine, CA: University of California, Irvine.

Hu, X., \& Batchelder, W. H. (1994). The statistical analysis of general processing tree models with the EM algorithm. Psychometrika, 59, 21-48.

JACOBY, L. L. (1991). A process dissociation framework: Separating automatic from intentional uses of memory. Journal of Memory \& Language, 30, 513-541

JENNINGS, J. M., \& JACOBY, L. L. (1993). Automatic versus intentional uses of memory: Aging, attention, and control. Psychology \& Aging, 8, 283-293.

Johnson, M. K., Hashtroudi, S., \& LindSay, D. S. (1993). Source monitoring. Psychological Bulletin, 114, 3-28.

Johnson, M. K., Kounios, J., \& ReEder, J. A. (1994). Time-course studies of reality monitoring and recognition. Journal of Experimental Psychology: Learning, Memory, \& Cognition, 20, 1409-1419.

JONES, G.V. (1987). Independence and exclusivity among psychological processes: Implications for the structure of recall. Psychological Review, 94, 229-235.

JOORDENS, S., \& MERIKLE, P. M. (1993). Independence or redundancy? Two models of conscious and unconscious influences. Journal of Experimental Psychology: General, 122, 462-467. 
Komatsu, S.-1., Graf, P., \& UtTL, B. (1995). Process-dissociation procedure: Core assumptions fail, sometimes. European Journal of Cognitive Psychology, 7, 19-40.

LECOMPTE, D. C. (1995). Recollective experience in the revelation effect: Separating the contributions of recollection and familiarity. Memory \& Cognition, 23, 324-334.

LiNDSAY, D. S., \& JACOBY, L. L. (1994). Stroop process dissociations: The relationship between facilitation and interference. Journal of Experimental Psychology: Human Perception \& Performance, 20, 219-234.

Richardson-Klavehn, A., Gardiner, J. M., \& JaVa, R. I. (1995). Memory: Task dissociations, process dissociations, and dissociations of consciousness. In G. Underwood (Ed.), Implicit cognition (pp. 85158). Oxford: Oxford University Press.

Riefer, D. M., \& BATChelder, W. H. (1988). Multinomial modeling and the measurement of cognitive processes. Psychological Review, 95, 318-339.

Riefer, D. M., Hu, X., \& BatChelder, W. H. (1994). Response strategies in source monitoring. Journal of Experimental Psychology: Learning, Memory, \& Cognition, 20, 680-693.

RoEDIGER, H. L., III, \& MCDERMOTT, K, B. (1994). The problem of differing false-alarm rates for the process dissociation procedure: Comment on Verfaellie and Treadwell (1993). Neuropsychology, 8, 284-288.

Toth, J. P., Reingold, E. M., \& JACOBY, L. L. (1994). Toward a redefinition of implicit memory: Process dissociations following elaborative processing and self-generation. Journal of Experimental Psychology: Learning, Memory, \& Cognition, 20, 290-303.

VATERRODT-PLÜNNECKE, B. (1994). Multinomiale Modellierung impliziter Gedächtnisprozesse: Ein alternativer Ansatz [Multinomial modeling of implicit memory processes: An alternative approach]. Zeitschrift für experimentelle und angewandte Psychologie, 41, 295-314.

Verfaellie, M., \& Treadwell, J. R. (1993). Status of recognition memory in amnesia. Neuropsychology, 7, 5-13.

WiPPICH, W. (1994). Unbewußte Effekte und Voreingenommenheiten bei Urteilen zu Personennamen [Unconscious effects and preconceptions in judgments of person names]. Zeitschrift für experimentelle und angewandte Psychologie, 41, 154-172.

Yonelinas, A. P. (1994). Receiver-operating characteristics in recognition memory: Evidence for a dual-process model. Journal of Experimental Psychology: Learning, Memory, \& Cognition, 20, 1341-1354.

Yonelinas, A. P., \& JACOBY, L. L. (1995). Dissociating automatic and controlled processes in a memory search task: Beyond implicit memory. Psychological Research, 57, 156-165.

\section{NOTES}

1. Technically $u_{\mathrm{c}+}$ is a nonidentifiable parameter, which means that an assumption must be made about this parameter in order to render the model identifiable (e.g., $u_{\mathrm{c}^{+}}=u_{\mathrm{c}-}, u_{\mathrm{c}+}=1$, or $u_{\mathrm{ct}}=0$, which yield, respectively, model variants in which controlled and automatic processes are independent, redundant, or mutually exclusive; see Jones, 1987). Which assumption is used, however, is relevant only if one is interested in the unconditional probability $u$ of automatic processes contributing to the recognition judgments. We suggest instead the use of $u_{\mathrm{c}-}$, which is the conditional probability of uncontrolled memory processes contributing to recognition, given that a Phase 1 item has not been recollected. This parameter is uniquely determined by the subjects' response probabilities and does not depend on which assumption is made about $u_{\mathrm{c}+}$. For details, see Buchner et al. (1995).

2. The power calculations were conducted using the G•Power program (Buchner, Faul, \& Erdfelder, 1992).

3. The log-likelihood goodness-of-fit statistic $G^{2}$ is asymptotically chi-square distributed with degrees of freedom indicated in parentheses (see Hu \& Batchelder, 1994, for details). All model-based statistical analyses reported in this article were conducted using the MBT program by Hu (1993; see Hu \& Batchelder, 1994).

4. As a supplement to these tests of the equality of memory processes, we analyzed whether models with the equality restriction imposed only on $c$ or on $u_{c-}$ would also fit the data. This was the case for both the read words $\left[G^{2}(1)=0.11\right.$ and $G^{2}(1)=0.96$ for the restriction on $c$ and $u_{c-}$, respectively] and the anagram words $\left[G^{2}(1)=3.78\right.$ and $G^{2}(1)=0.49$ for the restriction on $c$ and $u_{\mathrm{c}-}$, respectively]. Given the considerations explicated in the design section, we used $\chi^{2}(1)=$ $8.6398, \alpha=.0033$ as the critical value for these tests.

\section{APPENDIX}

Table A1

Frequencies of Phase 1, Phase 2, and New Responses for the Source-Monitoring Groups as a Function of Condition

\begin{tabular}{|c|c|c|c|c|c|c|}
\hline \multirow{4}{*}{$\begin{array}{c}\text { Participants' } \\
\text { Response }\end{array}$} & \multicolumn{6}{|c|}{ Source } \\
\hline & \multicolumn{3}{|c|}{ Inclusion } & \multicolumn{3}{|c|}{ Exclusion } \\
\hline & \multicolumn{2}{|c|}{$\begin{array}{l}\text { Phase } 1 \\
\text { Word }\end{array}$} & \multirow{2}{*}{$\begin{array}{l}\text { Distractor } \\
\text { Word }\end{array}$} & \multicolumn{2}{|c|}{$\begin{array}{l}\text { Phase } 1 \\
\text { Word }\end{array}$} & \multirow{2}{*}{$\begin{array}{l}\text { Distractor } \\
\text { Word }\end{array}$} \\
\hline & Read & Anagram & & Read & Anagram & \\
\hline Phase 1 & 131 & 292 & 48 & 150 & 270 & 78 \\
\hline Phase 2 & 80 & 47 & 28 & 69 & 51 & 29 \\
\hline New & 189 & 61 & 324 & 181 & 79 & 293 \\
\hline
\end{tabular}

(Manuscript received August 29, 1995; revision accepted for publication March 24, 1996.) 